\title{
OLD RECURRENCE FORMULAE FOR GROWTH SERIES OF COXETER GROUPS
}

\author{
JAN DYMARA (WROCŁAW)
}

\begin{abstract}
Several classical formulae for the growth series of a Coxeter group are proved in a new way, using the structure of the Coxeter complex, the Davis complex, or the Tits non-complex.
\end{abstract}

\section{INTRODUCTION}

Throughout this paper $(W, S)$ is a Coxeter system. This means that $W$ is the group generated by the finite set $S$, subject to relations of the form $(s t)^{m_{s t}}=1$, where $m_{s s}=1$ and for $t \neq s$ we have $m_{s t}=m_{t s} \in\{2,3, \ldots, \infty\}$ $(\infty$ means no relation). We will usually say "Coxeter group $W$ " instead of mentioning the whole system. We denote by $\ell(w)$ the word length of an element $w \in W$ with respect to the generating set $S$. For any subset $T \subseteq S$ the subgroup $W_{T}$ of $W$ generated by $T$ is also a Coxeter group, with relations being the relevant relations of $W$. The word length in $W_{T}$ agrees with the restriction to $W_{T}$ of the word length of $W$; we denote both by $\ell$. The growth series of $W$ is the formal power series $W(t)=\sum_{w \in W} t^{\ell(w)}$. We will prove several well-known formulae for this function. For infinite $W$ :

$$
\sum_{T \subseteq S} \frac{(-1)^{|T|}}{W_{T}(t)}=0
$$

If $W$ is finite, it has a unique element of longest length $m$. Then

$$
\sum_{T \subseteq S} \frac{(-1)^{|T|}}{W_{T}(t)}=\frac{t^{m}}{W(t)}
$$

A subset $T \subseteq S$ is called spherical if $W_{T}$ is finite. We denote by $\mathcal{S}$ the set of all spherical subsets of $S$. Let $\chi_{T}=\sum_{T \subseteq U \in \mathcal{S}}(-1)^{|U|}$. Then

$$
\begin{aligned}
& \sum_{T \in \mathcal{S}} \frac{(-1)^{|T|} \chi_{T}}{W_{T}(t)}=\frac{1}{W(t)} ; \\
& \sum_{T \in \mathcal{S}} \frac{(-1)^{|T|}}{W_{T}(t)}=\frac{1}{W\left(t^{-1}\right)} .
\end{aligned}
$$

2010 Mathematics Subject Classification. Primary 20F55; Secondary 57M07.

Key words and phrases. Coxeter group, growth series. 
These formulae are classical and quite popular, cf. [Bou, Ex. IV.26], [D, Chapter Seventeen], [ChD]; variants of Coxeter group growth series are investigated even nowadays, cf. [OS], [BGM, Prop. 8.3]. The usual proofs use an inclusion-exclusion principle for appropriate subsets of $W$, or induction on the cardinality of $S$. Our goal is to use spaces on which $W$ acts to establish (1), (2), (3) and (4) in an almost uniform way.

\section{Some properties of Coxeter groups}

The basic reference is [Bou], a newer one [D].

- For any $T \subseteq S$, and any $w \in W$, the coset $w W_{T}$ has a unique shortest element, say $u$. Moreover, if $w=u w^{\prime}$, then $\ell(w)=\ell(u)+\ell\left(w^{\prime}\right)$. ([D, Lemma 4.3.1])

- If $W$ is finite, then it has a unique element $w_{0}$ of longest length $m=\ell\left(w_{0}\right)$. Then, for any $w \in W$ we have $\ell(w)+\ell\left(w^{-1} w_{0}\right)=\ell\left(w_{0}\right)$ ([D, Lemma 4.6.1]). Consequently, in $W$ there are as many elements of length $k$ as of length $m-k$. More succinctly, $W(t)=t^{m} W\left(t^{-1}\right)$.

- For any $w \in W$, the set $\operatorname{In}(w)=\{s \in S \mid \ell(w s)<\ell(w)\}$ is spherical ([D, Lemma 4.7.2]).

\section{BASIC SPACES}

The basic construction ([D], Chapter Five) is performed as follows. Let $Y$ be a space (topological, or a simplicial complex) with a collection of subspaces $\left(Y_{s}\right)_{s \in S}$ (called panels). For $y \in Y$ we put $S(y)=\left\{s \in S \mid y \in Y_{s}\right\}$. Then $\mathcal{U}(W, Y)=W \times Y / \sim$, where $(w, y) \sim\left(w^{\prime}, y^{\prime}\right) \Longleftrightarrow y=y^{\prime}$ and $w^{-1} w^{\prime} \in W_{S(y)}$. The image of $\{w\} \times Y$ in $\mathcal{U}(W, Y)$ will be called a chamber and denoted $w Y$. The set of all chambers, $\operatorname{Ch}(\mathcal{U}(W, Y))$, is in bijective correspondence with $W$, provided $Y \neq \bigcup_{s \in S} Y_{s}$. Via this bijection the length function is transported to the set of chambers: $\ell(w Y)=\ell(w)$. The $W$ action on $\mathcal{U}(W, Y)$ is defined by $w(u, y)=(w u, y)$. For $T \subseteq S$ we put $Y_{T}=\bigcap_{t \in T} Y_{t}$ (we call it the face of type $T$ ) and $Y^{T}=\bigcup_{t \in T} Y_{t}$; this notation and terminology equivariantly extends to all chambers. We will use the notation $\sigma<X$ to indicate that $\sigma$ is a simplex of a simplicial complex $X$.

We will use three spaces arising from this construction.

1. The Coxeter complex $X_{\Delta}=\mathcal{U}(W, \Delta)$, where $\Delta$ is an $(|S|-1)$ dimensional simplex, and $\left(\Delta_{s}\right)_{s \in S}$ is the collection of its codimension-1 faces.

2. The Davis complex $X_{D}=\mathcal{U}(W, D)$. The chamber model $D$ (the Davis chamber) is the subcomplex of the first barycentric subdivision $\Delta^{\prime}$ of $\Delta$ spanned by the barycentres of faces of $\Delta$ that are of spherical type (for 
finite $W$ one should include also the barycentre of the empty face - we shall not consider this case). Alternatively, it is the subcomplex $X_{D}$ of the first barycentric subdivision $X_{\Delta}^{\prime}$ spanned by barycentres of simplices of $X_{\Delta}$ that are contained in finitely many chambers. It is a locally finite complex. The panels are $D_{s}=D \cap \Delta_{s}$, and the faces $D_{T}=D \cap \Delta_{T}$. An important property: for spherical $T$ the face $D_{T}$ is contractible; indeed, it is a cone with apex at the barycentre of $\Delta_{T}$. For non-spherical $T$ we have $D_{T}=\emptyset$. For any simplex $\sigma<D$ we define its type $S(\sigma)=\left\{s \in S \mid \sigma<D_{s}\right\}$; this definition extends by invariance to all simplices in $X_{D}$.

3. The Tits non-complex $X_{\Delta}^{f}=\mathcal{U}\left(W, \Delta^{f}\right)$. Here $\Delta^{f}$ is obtained from $\Delta$ by removing the closed simplices whose types are non-spherical. It is not a simplicial complex. For faces $\sigma$ of $\Delta$ of spherical type we still use the notation $\sigma<\Delta^{f}$, even though, in the geometric realisation, some part of $\sigma$ may not be a subset of $\Delta^{f}$.

For infinite $W$, all of the above spaces are contractible; $X_{D}$ and $X_{\Delta}^{f}$ even carry good CAT(0) metrics. Though they are generally very useful, these properties will not be relied upon in this note.

\section{Euler characteristic Generating FunCtion}

Let $X$ be either the Coxeter complex or the Davis complex for the Coxeter system $(W, S)$. (The case of the Tits non-complex is slightly different and will be dealt with in the last section.) For a simplex $\sigma<X$ we define its length $\ell(\sigma)$ as the minimum of $\ell(C)$, where $C$ runs through the chambers of $X$ that contain $\sigma$. There are finitely many chambers $C$ of $X$ of any given length, and each of them contains finitely many simplices. Therefore the following formal power series - the Euler characteristic generating function of $X$-is well defined:

$$
\chi_{t}(X)=\sum_{\sigma<X}(-1)^{\operatorname{dim} \sigma} t^{\ell(\sigma)} .
$$

We will calculate this series in two ways: grouping simplices according to type, and by local summation in $X$. Comparing the results we will obtain the formulae (1),(2) and (3).

\section{Summation according to type}

We consider $X=\mathcal{U}(W, Y)$ for $Y=\Delta$ or $D$. Let $\sigma<X$ be a simplex of type $T$. Then the chambers $C$ that contain $\sigma$ correspond to elements of some coset $u W_{T}$. Assume that $u$ is the shortest element in that coset. Then 
$\ell(\sigma)=\ell(u)$, and

$$
\sum_{C>\sigma} t^{\ell(C)}=\sum_{w \in u W_{T}} t^{\ell(w)}=\sum_{v \in W_{T}} t^{\ell(u)} t^{\ell(v)}=t^{\ell(u)} W_{T}(t)=t^{\ell(\sigma)} W_{T}(t) .
$$

Multiplying both sides by $(-1)^{\operatorname{dim} \sigma}$ and summing over all simplices of type $T$ we get

$$
\sum_{C \in \operatorname{Ch}(X)} t^{\ell(C)} \sum_{\substack{\sigma<C \\ S(\sigma)=T}}(-1)^{\operatorname{dim} \sigma}=W_{T}(t) \sum_{\substack{\sigma<X \\ S(\sigma)=T}}(-1)^{\operatorname{dim} \sigma} t^{\ell(\sigma)},
$$

which implies

$$
\frac{W(t)}{W_{T}(t)} \sum_{\substack{\sigma<Y \\ S(\sigma)=T}}(-1)^{\operatorname{dim} \sigma}=\sum_{\substack{\sigma<X \\ S(\sigma)=T}}(-1)^{\operatorname{dim} \sigma} t^{\ell(\sigma)} .
$$

Finally, we sum over all $T \subseteq S$ :

$$
W(t) \sum_{T \subseteq S} \frac{1}{W_{T}(t)} \sum_{\substack{\sigma<Y \\ S(\sigma)=T}}(-1)^{\operatorname{dim} \sigma}=\chi_{t}(X) .
$$

We will calculate the inner sum separately in each case.

The Coxeter complex. For any proper subset $T \subset S$ there is one $\sigma<\Delta$ of type $T$, namely $\Delta_{T}$. It has dimension $|S|-|T|-1$. Thus

$$
\chi_{t}\left(X_{\Delta}\right)=W(t) \sum_{T \subset S} \frac{(-1)^{|S|-|T|-1}}{W_{T}(t)} .
$$

The Davis complex. Recall that the face $D_{T}$ is a cone with apex at the barycentre of $\Delta_{T}$; let $L_{T}^{\prime}$ be its base $D_{T} \cap \partial \Delta_{T}$. The simplices $\sigma<D$ of type $T$ are the interior simplices of this cone (including the apex), so that

$$
\sum_{\substack{\sigma<D \\ S(\sigma)=T}}(-1)^{\operatorname{dim} \sigma}=1-\chi\left(L_{T}^{\prime}\right)
$$

Simplices in $L_{T}^{\prime}$ correspond to chains $U_{1} \subset \ldots \subset U_{k}$, where $U_{i}$ are spherical and properly contain $T$. This means that $L_{T}^{\prime}$ is, as the notation suggests, the barycentric subdivision of the following simplicial complex $L_{T}$. The vertices of $L_{T}$ correspond to spherical sets of the form $T \cup\{u\}$ (with $u \in S-T$ ); vertices $T \cup\left\{u_{1}\right\}, \ldots, T \cup\left\{u_{k}\right\}$ span a simplex if $T \cup\left\{u_{1}, \ldots, u_{k}\right\}$ is spherical. Thus, simplices of $L_{T}$ correspond to spherical $U$ containing $T$, and

$$
1-\chi\left(L_{T}^{\prime}\right)=1-\chi\left(L_{T}\right)=\sum_{T \subseteq U \in \mathcal{S}}(-1)^{|U|-|T|}=(-1)^{|T|} \chi_{T}
$$

for $\chi_{T}=\sum_{T \subseteq U \in \mathcal{S}}(-1)^{|U|}$. Finally, returning with this to (9) we get

$$
\chi_{t}\left(X_{D}\right)=W(t) \sum_{T \in \mathcal{S}} \frac{(-1)^{|T|} \chi_{T}}{W_{T}(t)} .
$$




\section{LOCAL SUMMATION}

We continue with the cases $Y=\Delta$ or $D$. Now we compute the sum $\sum_{\sigma<X}(-1)^{\operatorname{dim} \sigma} t^{\ell(\sigma)}$ by arranging its summands according to chambers: we consider each $\sigma$ as part of the chamber realising $\ell(\sigma)$. We get

$$
\chi_{t}(X)=\sum_{C \in \operatorname{Ch}(X)} t^{\ell(C)} \sum_{\substack{\sigma<C \\ \ell(\sigma)=\ell(C)}}(-1)^{\operatorname{dim} \sigma}=\sum_{w \in W} t^{\ell(w)} \sum_{\substack{\sigma<w Y \\ \ell(\sigma)=\ell(w)}}(-1)^{\operatorname{dim} \sigma}
$$

Lemma 6.1. Let $\sigma<w Y$. Then $\ell(\sigma)<\ell(w)$ if and only if $\sigma<(w Y)^{\operatorname{In}(w)}$.

Proof. Let $T$ be the type of $\sigma$. The chambers containing $\sigma$ correspond to elements of the coset $w W_{T}$.

Suppose $\ell(\sigma)<\ell(w)$. It follows that $w$ is not the shortest element of the coset $w W_{T}$. Therefore $\ell(w t)<\ell(w)$ for some $t \in T$. This $t$ also belongs to $\operatorname{In}(w)$. Finally, $\sigma<w Y \cap w t Y=(w Y)_{t}$.

Conversely, if $\sigma<(w Y)^{\operatorname{In}(w)}$, then $\sigma<(w Y)_{s}$ for some $s \in \operatorname{In}(w)$. But then $\sigma<w s Y$ and $\ell(\sigma) \leq \ell(w s)<\ell(w)$. The Lemma is proved.

By the Lemma, the inner sum in (14) is performed over simplices of $w Y$ that are not in $(w Y)^{\operatorname{In}(w)}$. Thus

$$
\begin{aligned}
\sum_{\substack{\sigma<w Y \\
\ell(\sigma)=\ell(w)}}(-1)^{\operatorname{dim} \sigma} & =\chi\left(w Y,(w Y)^{\operatorname{In}(w)}\right)=\chi\left(Y, Y^{\operatorname{In}(w)}\right) \\
& =\chi(Y)-\chi\left(Y^{\operatorname{In}(w)}\right)=1-\chi\left(Y^{\operatorname{In}(w)}\right),
\end{aligned}
$$

the last equality due to contractibility of $Y$.

We split the calculation of $\chi\left(Y^{\operatorname{In}(w)}\right)$ into three cases.

1) The generic case: $\operatorname{In}(w)$ is a proper non-empty subset of $S$, of some cardinality $k$. Notice that for any $T \subseteq \operatorname{In}(w)$ (a fortiori, spherical) the face $Y_{T}$ is contractible. Using the inclusion-exclusion principle we get

$$
\begin{gathered}
\chi\left(Y^{\operatorname{In}(w)}\right)=\sum_{s \in \operatorname{In}(w)} \chi\left(Y_{s}\right)-\sum_{\substack{s, t \in \operatorname{In}(w) \\
s \neq t}} \chi\left(Y_{\{s, t\}}\right)+\ldots+(-1)^{k-1} \chi\left(Y_{\operatorname{In}(w)}\right) \\
=k-\left(\begin{array}{l}
k \\
2
\end{array}\right)+\left(\begin{array}{l}
k \\
3
\end{array}\right)-\ldots+(-1)^{k-1}\left(\begin{array}{l}
k \\
k
\end{array}\right)=1-(1-1)^{k}=1 .
\end{gathered}
$$

2) The set $\operatorname{In}(w)$ is empty. Then $\chi\left(Y^{\operatorname{In}(w)}\right)=\chi(\emptyset)=0$. This happens exactly when $w=1$.

3) The case $\operatorname{In}(w)=S$. This happens only if $W$ is finite and $w$ is the element of longest length (say length $m$ ). Then $Y^{S}$ is a triangulation of the $(|S|-2)$-dimensional sphere, $\chi\left(Y^{S}\right)=1-(-1)^{|S|-1}$. 
Plugging these results into (15), and then into (14), we get for infinite $W$

$$
\chi_{t}\left(X_{\Delta}\right)=1, \quad \chi_{t}\left(X_{D}\right)=1,
$$

while for finite $W$ with element of longest length $m$

$$
\chi_{t}\left(X_{\Delta}\right)=1+(-1)^{|S|-1} t^{m} .
$$

Comparing these results with (10) and (13) we get (1), (2) and (3).

\section{The TITS NON-COMPLEX}

Consider now the case $X=X_{\Delta}^{f}$. For $\sigma<X$ we define $L(\sigma)$ as the maximum of $\ell(C)$ over all chambers containing $\sigma$. This is finite, since the type of $\sigma$ is spherical. Then we define $\chi^{t}(X)=\sum_{\sigma<X}(-1)^{\operatorname{dim} \sigma} t^{L(\sigma)}$. As before, we will calculate this sum in two ways.

First, we group the summands according to the type of $\sigma$. Let $\sigma<X$ be a simplex of (spherical!) type $T$. Then the chambers $C$ that contain $\sigma$ correspond to elements of some finite coset $u W_{T}$. Assume that $u$ is the longest element in that coset. Then $L(\sigma)=\ell(u)$, and

$$
\begin{aligned}
\sum_{C>\sigma} t^{\ell(C)}=\sum_{w \in u W_{T}} t^{\ell(w)}=\sum_{v \in W_{T}} t^{\ell(u)} t^{-\ell(v)} & =t^{\ell(u)} W_{T}\left(t^{-1}\right) \\
& =t^{L(\sigma)} W_{T}\left(t^{-1}\right) .
\end{aligned}
$$

Multiplying both sides by $(-1)^{\operatorname{dim} \sigma}\left(=(-1)^{|S|-|T|-1}\right)$ and summing over all simplices of type $T$ (there is just one in each chamber) we get

$$
(-1)^{|S|-|T|-1} \sum_{C \in \operatorname{Ch}(X)} t^{\ell(C)}=W_{T}\left(t^{-1}\right) \sum_{\substack{\sigma<X \\ S(\sigma)=T}}(-1)^{\operatorname{dim} \sigma} t^{L(\sigma)},
$$

which implies

$$
\frac{W(t)}{W_{T}\left(t^{-1}\right)}(-1)^{|S|-|T|-1}=\sum_{\substack{\sigma<X \\ S(\sigma)=T}}(-1)^{\operatorname{dim} \sigma} t^{L(\sigma)} .
$$

Summing this equality over all spherical types $T$ we get

$$
W(t) \sum_{T \in \mathcal{S}} \frac{(-1)^{|S|-|T|-1}}{W_{T}\left(t^{-1}\right)}=\chi^{t}\left(X_{\Delta}^{f}\right)
$$

Second, we group summands in chambers according to $L$ :

$$
\chi^{t}(X)=\sum_{\sigma<X}(-1)^{\operatorname{dim} \sigma} t^{L(\sigma)}=\sum_{w \in W} t^{\ell(w)} \sum_{\substack{\sigma<w \Delta f \\ L(\sigma)=\ell(w)}}(-1)^{\operatorname{dim} \sigma} .
$$


Every $\sigma<w \Delta^{f}$ is of the form $w \Delta_{T}$ for some spherical $T$; moreover, $L(\sigma)=$ $\ell(w)$ if and only if $w$ is the longest element in the coset $w W_{T}$, which happens exactly when $T \subseteq \operatorname{In}(w)$. Therefore

$$
\sum_{\substack{\sigma<w \Delta f \\ L(\sigma)=\ell(w)}}(-1)^{\operatorname{dim} \sigma}=\sum_{T \subseteq \operatorname{In}(w)}(-1)^{|S|-|T|-1}=(-1)^{|S|-1}(1-1)^{|\operatorname{In}(w)|} .
$$

Thus, the only non-zero summand corresponds to $w$ with empty $\operatorname{In}(w)$, that is to $w=1$. We get

$$
\chi^{t}\left(X_{\Delta}^{f}\right)=(-1)^{|S|-1} .
$$

Comparing (25) with (22), and switching $t$ and $t^{-1}$, we get (4).

Acknowledgements. The author was partially supported by the Polish National Science Centre (NCN) grant 2016/23/B/ST1/01556.

\section{REFERENCES}

[Bou] N. Bourbaki. Groupes et algèbres de Lie, Chapitres IV-VI. Masson, 2nd edition, 1981.

[BGM] M. Bożejko, Ś. Gal, W. Młotkowski. Positive definite functions on Coxeter groups with applications to operator spaces and noncommutative probability, Comm. Math. Phys. 361(2018), no. 2, 583-604.

[ChD] R. Charney, M. Davis. Reciprocity of growth functions of Coxeter groups. Geom. Dedicata 39(1991), no. 3, 373-378.

[D] M. Davis. The Geometry and Topology of Coxeter Groups. Princeton University Press, Princeton and Oxford 2008.

[OS] B. Okun, R. Scott. Growth series of CAT(0) cubical complexes, Topology Proc. 54(2019), 295-303.

Instytut Matematyczny

Uniwersytet Wrocławski

pl. Grunwaldzki 2/4, 50-384 Wrocław

Poland

dymara@math.uni.wroc.pl 